Pesq. Vet. Bras. 37(11):1336-1340, novembro 2017

DOI: $10.1590 / \mathrm{S} 0100-736 \mathrm{X} 2017001100023$

\title{
Fatores relacionados a problemas de comportamento em gatos ${ }^{1}$
}

\author{
Juliane E.G. Paz ${ }^{2}$, Gustavo Machado ${ }^{3}$ e Fernanda V. Amorim da Costa* ${ }^{4 *}$
}

\begin{abstract}
Paz J.E.G., Machado G. \& Costa F.V.A. 2017. [Factors associated with behavior problems in cats.] Fatores relacionados a problemas de comportamento em gatos. Pesquisa Veterinária Brasileira 37(11):1336-1340. Departamento de Medicina Animal, Faculdade de Veterinária, Universidade Federal do Rio Grande do Sul, Avenida Bento Gonçalves 9090, Porto Alegre, RS 91540-000, Brazil. E-mail: fernanda.amorim@ufrgs.br

Behavioral problems have importance not only in animal welfare and the quality of human-animal relationship, but also in public health. Behavior problems are a major reason of abandonment and subsequently the fate of these animals are shelters or even euthanasia. Furthermore, the aggressiveness is a risk factor for zoonoses transmission. In order to assess the frequency of factors related to behavior problems, information from 229 cats were collected through a questionnaire applied to tutors awaiting for care in clinics in Veterinary Hospital of the Federal University of Rio Grande do Sul (HCV-UFRGS). Among the behaviors scratch furniture was the most common problem (65.1\% - $\mathrm{CI}_{95 \%}: 58.5$ to $\left.71.4 \%\right)$, followed by aggressiveness $\left(61.3 \%-\mathrm{CI}_{95 \%}: 54.2\right.$ to $\left.67,1 \%\right)$, inappropriate elimination $\left(37.1 \%-9 \mathrm{CI}_{95 \%}\right.$ : 31.3 to $42.1 \%$ ) and excessive vocalization (23.6\% - $\mathrm{CI}_{95 \%}: 20.1$ to $\left.29.8 \%\right)$. Data were analyzed by logistic regression with a logit link function. Univariate analysis identified the presence of scratching as significant protective factor, that may contribute to the reduction of scratching furniture (Relative risk $[\mathrm{RR}]=0.31-\mathrm{CI}_{95 \%}: 0: 19$ to $0: 53$ ). Females and frightened cats were identified as having more difficulty were for friendly relationship with other cats $\mathrm{RR}=3.56$ $\left(\mathrm{CI}_{95 \%}\right.$ : 1.78-7.11), $\mathrm{RR}=2.84$ (95\% CI: 1.60-5.04) respectively; also the higher the number of cats in a residence, the greater the chance for inappropriate elimination. Neutered cats before puberty had less chance of developing inappropriate elimination $\mathrm{RR}=0: 43\left(\mathrm{CI}_{95 \%}: 0.21-0.88\right)$, and finally, excessive vocalization was related to obtain tutor attention $\mathrm{RR}=2.62\left(\mathrm{CI}_{95 \%}: 1: 54\right.$ to $4: 45$ ). The results of this study may help prevent behavioral problems in domestic cats.

INDEX TERMS: Cats, aggression, inappropriate elimination, scratching, behavioral problems.
\end{abstract}

RESUMO.- Os problemas de comportamento apresentam importância não só no bem estar animal e na qualidade da relação humana-animal como também em saúde publica. Problemas de comportamento são uma das principais razões de abandono e subsequentemente o destino destes animais são abrigos ou até mesmo eutanásia. Além disso, a agressividade é um fator de risco para transmissão de zoo-

\footnotetext{
${ }^{1}$ Recebido em 10 de junho de 2015.

Aceito para publicação em 13 de janeiro de 2017

${ }^{2}$ Médica Veterinária Autônoma, Rua Dário Pederneiras 577, Porto Alegre, RS 90630-090, Brasil.

${ }^{3}$ Laboratório de Epidemiologia Veterinária, Faculdade de Veterinária, Universidade Federal do Rio Grande do Sul, Av. Bento Gonçalves 9090, Porto Alegre, RS 91540-000, Brasil.

${ }^{4}$ Departamento de Medicina Animal, Faculdade de Veterinária, Universidade Federal do Rio Grande do Sul, Avenida Bento Gonçalves 9090, Bairro Agronomia, Porto Alegre, RS 91540-000, Brasil. *Autor para correspondência: fernanda.amorim@ufrgs.br
}

noses. Com o objetivo de avaliar a frequência de fatores relacionados a problemas de comportamento em gatos, foram coletadas informações de 229 gatos através de um questionário aplicado em tutores que aguardavam atendimento no Hospital de Clínicas Veterinária da Universidade Federal do Rio Grande do Sul (HCV-UFRGS). Dentre os comportamentos arranhadura em móveis foi o problema comportamental mais frequente $\left(65,1 \%\right.$ - IC $\left._{95 \%}: 58,5-71,4 \%\right)$ seguido de agressividade $\left(61,3 \%\right.$ - IC $\left.{ }_{95 \%}: 54,2-67,1 \%\right)$, eliminação inapropriada (37,1\%- IC $95 \%$ : 31,3-42,1\%) e vocalização excessiva $\left(23,6 \%-\mathrm{IC}_{95 \%}: 20,1-29,8 \%\right)$. Os dados foram analisados por regressão logística com uma função de ligação logit. De acordo com a análise univariada, a presença de um arranhador teve uma relação significativa protetiva, podendo contribuir para a redução do comportamento de arranhar móveis (risco relativo $[R R]=0.31-I_{95 \%}: 0.19-0.53$ ). Fêmeas e gatos assustados foram identificados com maior dificuldade de ter uma relação amigável com outros gatos $\mathrm{RR}=3.56$ (IC 
${ }_{95 \%}: 1.78$ - 7.11), RR=2.84( IC $_{95 \%}: 1.60$ - 5.04) respectivamente também quanto maior o número de gatos na residência, maiores as chances de haver eliminação inapropriada. Os gatos castrados antes da puberdade apresentaram menos chances de desenvolver eliminação inapropriada $\mathrm{RR}=0.43$ (IC $\left.{ }_{95 \%}: 0.21-0.88\right)$ e, finalmente, a vocalização excessiva foi relacionada com busca de atenção RR=2.62 (IC ${ }_{95 \%}: 1.54$ 4.45). Os resultados desse estudo podem ajudar a prevenir problemas comportamentais em felinos domésticos.

TERMOS DE INDEXAÇÃO: Felinos, agressividade, eliminação inapropriada, arranhaduras, problemas comportamentais.

\section{INTRODUÇÃO}

A relação entre felinos e humanos é bem antiga, porém mais recente que a dos cães, data-se de cerca de 10 mil anos atrás, dando-se através de uma relação de mutualismo. Os felinos eram atraídos por roedores que se alojavam em locais de armazenamento de grãos destinados à alimentação humana. Diferente do cão, a domesticação do gato não resultou, a princípio, em modificações do seu comportamento natural nem seleção genética (Overall 1997). Existe uma discussão de que os gatos teriam se "auto-domesticado", ou seja, que o homem não teve papel algum nas mudanças, exceto permitindo o convívio próximo dos gatos e fazendo com que aumentasse suas chances de sobrevivência e sucesso reprodutivo. Posteriormente, a influência do ser humano na domesticação dos gatos se deu de forma gradual e cada vez mais significativa (Price 1984). Por esses motivos, os gatos domésticos mantêm muitas características comportamentais de seus antecessores selvagens (Griffin \& Hume 2006).

A remanescência do perfil comportamental do gato doméstico com seu ancestral selvagem pode trazer algumas consequências indesejáveis para tutores. A definição mais apropriada de problema comportamental é qualquer comportamento apresentado pelo animal que é inaceitável para o tutor (Amat et al. 2009), sendo que alguns destes comportamentos são naturais do gato, como arranhadura de objetos e marcação do ambiente com urina, mas que, em casa, tornam-se indesejáveis (Cassey \& Bradshaw 2008). De acordo com Turner (1991), muitos problemas de comportamento resultam de uma falha ao considerar as necessidades do gato, as condições ambientais ou mudanças, as expectativas irreais do tutor ou da interação inadequada entre tutor e gato.

O desenvolvimento dos problemas de comportamento é uma das principais causas de abandono (Miller et al. 1996). Segundo Heath (2006) os problemas comportamentais foram responsáveis por 7\% dos casos de abandono em um abrigo de animais. Entre os problemas de comportamento a agressividade, arranhadura e eliminação inapropriada foram citadas como as razões mais frequentes para o abandono em uma pesquisa realizada na Universidade Federal Fluminense (Souza-Dantas et al. 2009).

Além disso, os problemas de comportamento podem ser uma ameaça à saúde pública. Em muitos estudos, a agressividade é um dos principais problemas relatados pelos tutores (Morgan \& Houpt 1990, Heidenberger 1997, Heath 2006, Amat et al. 2009). Os arranhões e mordidas causados por comportamentos agressivos são preocupantes do pelo risco de transmissão de zoonoses, principalmente doenças como raiva e bartonelose, além de interferir no bem-estar animal e na relação animal-tutor, que fica prejudicada (Rochlitz 2000).

0 presente estudo teve como objetivo verificar a frequência dos problemas comportamentais de felinos levados ao HCV-UFRGS para atendimento clínico e, além disso, identificar possíveis fatores relacionados ao surgimento de problemas comportamentais.

\section{MATERIAL E MÉTODOS}

Área e população estudada. Um questionário elaborado pelos autores foi aplicado a tutores de gatos que aguardavam atendimento no Hospital de Clínicas Veterinárias da UFRGS. Este hospital possui cerca de 5.000 atendimentos a gatos por ano e recebe clientes de diversas partes de Porto Alegre e cidades próximas. Através do questionário, coletou-se informações sobre o tutor, o animal, o ambiente em que os gatos viviam e informações a respeito da ocorrência de problemas comportamentais (Quadro 1). O questionário foi validado semanticamente, a fim de evitar que uma assertiva pudesse ter mais de uma interpretação.

\section{Quadro 1. Descrição das variáveis coletadas por meio de} questionário epidemiológico

\begin{tabular}{ll}
\hline Variáveis dependentes & \multicolumn{1}{c}{ Variáveis independentes } \\
\hline Agressividade e motivo & Idade do tutor e do animal \\
Relação com outros animais & Escolaridade \\
Vocalização excessiva & Raça \\
Arranhadura em móveis & Sexo \\
Eliminação inapropriada & Pelagem \\
& Cor \\
& Residência \\
& Acesso à rua \\
& Experiência previa do tutor com gatos \\
& Origem do gato \\
& Idade ao adquirir \\
& Idade ao castrar \\
& Animais coabitantes \\
& Local e número de vasilhas sanitárias \\
& Tipo de substrato da vasilha \\
& Presença de crianças em casa \\
& Grau de atividade do gato \\
& Presença de arranhador \\
& Tempo que o gato fica sozinho e importa-se \\
& ou não de ficar sozinho \\
& Nível de afetividade do tutor com o animal \\
& Características comportamentais do gato \\
(ex. tímido, curioso) & Motivo da ida ao veterinário \\
Histórico de doenças &
\end{tabular}

Amostragem e obtenção das amostras. Primeiramente, um estudo seccional foi realizado para a obtenção de informações relacionadas a problemas de comportamento em felinos. Para obtenção e seleção dos participantes foram utilizados os seguintes parâmetros amostrais: magnitude de dois do desfecho (alterações de comportamento) com $80 \%$ de poder com nível de confiança de 95\% (Kelsey et al. 1996):

$$
\mathrm{n}_{1}=\frac{\left(\mathrm{Z}_{\alpha / 2}+\mathrm{Z}_{1}-\beta\right)^{2} \overline{\mathrm{p}}-\overline{\mathrm{q}}(\mathrm{r}+1)}{\mathrm{r}\left(\mathrm{p}_{1}-\mathrm{P}_{1}\right)^{2}}
$$

$\mathrm{e}$

$$
\mathrm{n}_{2}=\mathrm{rn}_{1}
$$

Onde $n_{1}=$ número de expostos; $n_{2}=$ número de não-expostos; $\mathrm{Z}_{\alpha / 2}=$ desvio padrão para teste de dupla cauda, baseada em alfa 
de $10 \% ; Z_{\beta}=$ desvio padrão para teste de dupla cauda, baseada em beta; $r=$ risco dos não-exposto em relação aos expostos; $\mathrm{p}_{1}=$ proporção de expostos com o desfecho e $\mathrm{q}_{1}=1-\mathrm{p}_{1}, \mathrm{p}_{2}=$ proporção de expostos sem o desfecho $\mathrm{q}_{2}=1-\mathrm{p}_{2}$ Com base na metodologia acima o número mínimo de tutores a serem entrevistados foi de 212 . No presente estudo foram aplicados 229 questionários, e, ainda como critério de inclusão no estudo, somente tutores portando felinos com idade superior a cinco meses foram amostrados. $\mathrm{O}$ questionário foi aplicado em todos os tutores que aguardavam atendimento tanto clínico geral como nas especialidades diversas e não apenas tutores que procuravam atendimento comportamental. Todos os tutores participantes assinaram termo de concordância em participar do estudo e para a publicação dos dados obtidos.

Análise estatística. Os dados foram armazenamos em planilhas Excel e analisados por meio de estatística descritiva, distribuição de frequências, construção de tabelas de contingência e regressão logística. Como variáveis dependentes foram analisadas: agressividade, relação com outros animais, vocalização excessiva, arranhadura em móveis e eliminação inapropriada (Quadro 1). Foram construídos cinco modelos de regressão logística, um para cada variável dependente, sendo ofertadas para os modelos de regressão todas as variáveis independentes conforme Quadro 1. Variáveis com dados faltantes $(>10 \%)$ e com variabilidade limitada $(<20 \%)$ foram excluídas para as análises de regressão. A análise univariada foi realizada com o teste do qui-quadrado de Pearson $\left(\chi^{2}\right)$, enquanto que um teste do qui-quadrado para tendências lineares ou teste exato de Fisher para examinar a associação entre variáveis dependentes e variáveis independente. 0 risco relativo foi calculado para avaliar o impacto das variáveis independentes no comportamento estudado, considerando diferença significativa $\mathrm{P}<0.05$. Todas as análises foram realizadas na linguagem $\mathrm{R}$ v.2.15.2 (R development Core Team $^{\circledR}, 2012$; packagedEpiCalc). 0 estudo foi aprovado pelo Comitê de Ética em Pesquisa da Universidade Federal do Rio Grande do Sul e devidamente cadastrado na Plataforma Brasil sob o parecer número 336.239.

\section{RESULTADOS}

O questionário foi aplicado em 229 tutores, sendo a maioria mulheres com idade média de 44,5 anos. Os gatos possuíam média de idade de 6,2 anos. A maioria dos gatos que participaram da pesquisa eram fêmeas, correspondendo a $52 \%$ dos gatos analisados, e sem raça definida ( $79 \%$ dos gatos). A arranhadura em móveis foi o problema comportamental mais frequente (65\%- IC ${ }_{95 \%}$ : 58,5-71,4\%) na população de gatos analisada, seguida por agressividade $\left(61,1 \%\right.$ - IC ${ }_{950 \%}$ : $54,2-67,1 \%)$, eliminação inapropriada $(37,1 \%$ - IC 950 : $31,3-$ $42,1 \%)$ e vocalização excessiva (23,6\%- IC ${ }_{95 \%}$ : $\left.20,1-29,8 \%\right)$.

A arranhadura em móveis teve associação com ausência de local destinado para este fim, gatos que tinham um arranhador ou local próprio para arranhar apresentaram 69\% menos risco de arranhar móveis, o que está demonstrado no Quadro 2. Sexo e o fato de ser considerado assustado pelo tutor foram relevantes na influência da relação entre os gatos. Gatos assustados apresentaram 3,5 vezes mais chances de terem uma relação ruim ou indiferente com outros gatos e fêmeas 2,8 vezes mais chances, como indica o Quadro 2.

Quanto à eliminação inapropriada, animais castrados antes da puberdade apresentaram $57 \%$ menos chance de desenvolverem o problema quando comparados a gatos castrados após a puberdade. 0 número de gatos na casa também influenciou esse problema, sendo que gatos que
Quadro 2. Regressão logística univariada, fatores associados a problemas de comportamento

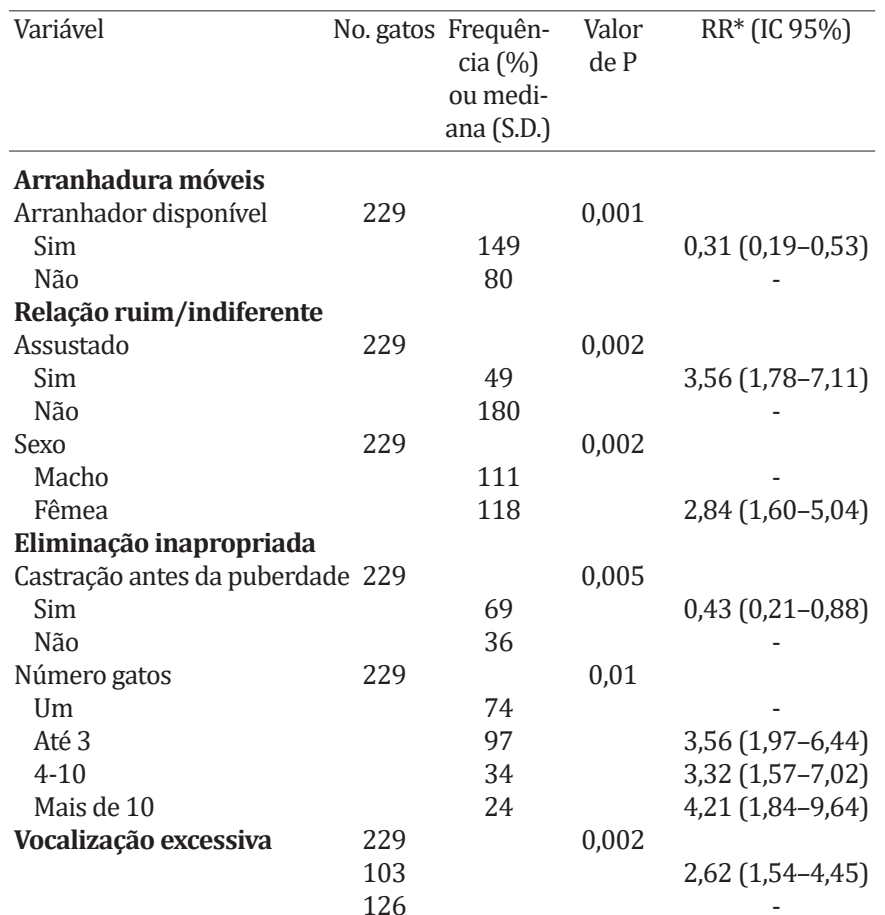

*Risco relativo

residem em ambientes com mais de dez gatos apresentam um risco 4,2 maior de sofrer esse problema (Quadro 2).

Tutores de 54 animais afirmaram que havia vocalização excessiva, os animais miavam sem motivo aparente. A vocalização excessiva foi relacionada com animais caracterizados pelos tutores como gatos que buscavam atenção, demostrado no Quadro 2.

A agressividade teve uma frequência bastante elevada, 27 gatos apresentaram agressividade contra animais, sendo 16 destes, agressividade intraespecífica. A agressividade contra pessoas foi relatada em 55 gatos, sendo na maioria das vezes motivada por carinho (40\%) e em segundo lugar por brincadeira (36,3\%) (Fig.1). Relatou-se em 58 gatos agressividade tanto contra pessoa como contra animais. Nenhum fator foi estatisticamente relacionado com agressividade.

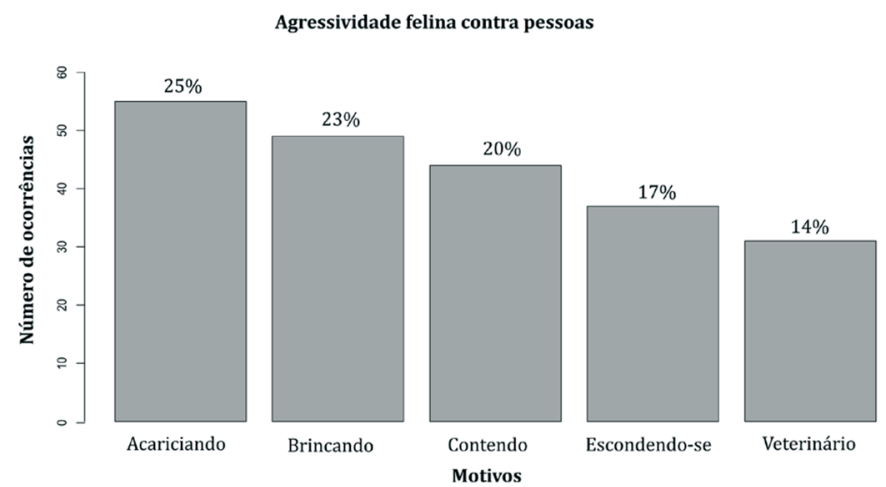

Fig.1. Frequência das agressividades quando solicitado ao tutor responder se o gato costumava ser agressivo nas seguintes situações: quando acariciado, quando estava brincando, quando carregado no colo, quando estava escondido e quando ia ao veterinário. 


\section{DISCUSSÃO}

O problema de comportamento mais frequente na população de gatos estudada foi arranhadura de móveis, seguido de agressividade, eliminação inapropriada e vocalização excessiva. Esse resultado é bastante semelhante ao encontrado por Morgan \& Houpt (1990), onde arranhadura em móveis também foi o problema mais comum incluindo $60 \%$ dos casos, seguido de agressividade com $36 \%$, eliminação inapropriada e vocalização com $16 \%$. Outros estudos encontram eliminação inapropriada como principal problema de comportamento (Borchelt \& Voith 1986, Fatjó et al. 2006, Souza-Dantas et al. 2009, Wassink-Van der Schot et al. 2016). Já Amat et al. (2009) encontrou agressividade como sendo o mais comum.

A arranhadura é um comportamento inato dos felinos que não deve ser combatido, mas sim redirecionado para locais adequados. Esse comportamento tem dois propósitos: criar uma marcação visual e fazer a remoção das bainhas velhas. Por conta das glândulas presentes nas mãos dos gatos esse comportamento também deixa uma marcação olfativa que tem a função de passar segurança ao gato residente (Beaver 2003). A presença de local próprio para arranhar representou um menor risco dos gatos arranharem móveis. Esse resultado reforça a importância do tutor fornecer um local próprio para o gato arranhar desde jovem, pois uma vez que o gato escolha um local para arranhar ele continuará usando esse local durante toda sua vida (Marder 1997). A escolha do local adequado também é importante, já que a maioria dos gatos prefere desempenhar esse comportamento em locais próximos das áreas de descanso e áreas de entrada e saída da casa (Case 2010). Além de fornecer arranhadores, é preciso observar as preferências específicas do gato, pois alguns preferem arranhar na posição vertical e outros na horizontal (Overall 1997).

Quanto à relação entre os animais, fatores que influenciaram para uma relação ruim ou indiferente foram o sexo feminino e gatos considerados assustados pelos tutores. Esses apresentaram maior chance de não ter uma relação amigável com outros animais. Um estudo diagnosticou agressividade por medo como sendo a causa mais comum de agressividade entre gatos, principalmente quando há mudanças nos grupos de gatos de uma casa (Borchelt \& Voith 1987). Esse resultado pode estar relacionado ao que foi encontrado no presente estudo, sugerindo que gatos assustados possam atacar outros gatos por medo.

Os fatores que interferem na relação entre os gatos têm sido bastante estudados. Uma pesquisa realizada com gatos ferais sugere que eles são mais propensos a se aproximarem do gênero oposto (Wolfe, Sung \& Crowell-davis 1997). Entretanto, Barry e Crowell-Davis (1999) não encontraram diferenças significativas no relacionamento entre os gêneros de gatos castrados sem acesso à rua (Barry \& Crowell-Davis 1999). 0 presente estudo sugere que as fêmeas são mais propícias a terem uma relação ruim ou indiferente com outros gatos. Wassink-Van der Schot (2016) encontrou um resultado semelhante onde a agressividade entre gatos familiares era mais frequente em gatas fêmeas.

A maior parte dos casos de agressividade foi voltada contra pessoas. Fatjó et al. (2006) e Wassink-Van der Schot
(2016) encontraram o mesmo resultado. Entretanto, outros estudos encontraram resultados opostos com tutores que afirmaram que os gatos eram mais propensos a serem agressivos com outros gatos do que com pessoas (Borchelt \& Voith 1996, Tamimi et al. 2015). Também Amat et al. (2009) encontrou maior porcentagem de agressividade intraespecífica (64\%) do que contra pessoas (36\%). Uma possibilidade para essa discrepância é que uma parte considerável dos tutores entrevistados (32\%) possuía apenas um gato na residência. Esse fator pode ter superestimado os casos de agressividade contra pessoas. Segundo Ramos \& Mills (2009), a ausência de outros gatos representa um aumento de risco para agressividade contra pessoas. É importante considerar também que tem sido reportado que muitos tutores consideram a agressividade como um comportamento normal e o toleram (Borchelt \& Voith 1987).

Beaver (2003) afirma em seu estudo que a eliminação inapropriada é o problema de comportamento mais comum em gatos, já que cerca de $50 \%$ dos tutores reclamam desse problema. Na população estudada, a eliminação inapropriada foi o terceiro comportamento mais frequente. 0 número de gatos na residência interferiu na frequência desse problema. Em ambientes com até três gatos, houve cerca de três vezes mais chance do desenvolvimento desse problema, e em ambientes com mais de dez, cerca de quatro vezes mais chance. Esse fator talvez esteja relacionado com a dificuldade de fornecer o número adequado de vasilhas sanitárias para o número de gatos. 0 número adequado de bandejas de areia é igual ao número de gatos mais uma extra (Houpt 1985). As interações sociais também devem ser consideradas e podem ser fatores predisponentes para a eliminação inapropriada. Um gato pode evitar usar a vasilha sanitária se ele é atacado por outro gato quando vai usá-la ou se é encurralado dentro dela depois de usá-la podendo assim escolher outro local mais seguro para urinar e defecar. Um gato que vive em um ambiente hostil pode marcar o local com urina secundariamente a questões territoriais ou de ansiedade (Neilson 2004). Quanto maior o número de gatos, maiores as chances de surgirem interações sociais negativas.

Outro fator que teve relação com eliminação inapropriada foi a idade ao castrar. Animais castrados antes da puberdade tiveram menor chance de apresentar esse comportamento. Alguns estudos já têm demonstrado que a castração elimina esse problema na maioria dos gatos (Hart \& Barret 1973, Hart \& Cooper 1984, Calixto \& Justen 2007). Animais castrados após a puberdade tendem a manter o comportamento já que acabam criando o hábito de marcar território.

A vocalização excessiva pode estar relacionada a alguns problemas de saúde, entretanto, esta também pode ser aprendida através de reforço positivo (Overall 1997, Beaver 2003). No presente estudo, a vocalização excessiva foi relacionada a gatos que buscavam atenção dos tutores. Esse resultado sugere que esse comportamento pode ser reforçado pelo próprio tutor. Um estudo encontrou que gatos mais velhos são mais propensos a buscarem atenção dos tutores e que fêmeas inteiras parecem procurar menos atenção (Tamimi et al. 2015). No presente estudo não houve relação entre busca de atenção e idade ou sexo. 


\section{CONCLUSÕES}

Sugere-se que alguns problemas de comportamento podem ser evitados, através do fornecimento de um local próprio para o gato arranhar, evitando a arranhadura em móveis, da realização da castração antes da puberdade, bem como evitando o acúmulo de muitos gatos em um recinto, reduzindo desta forma o risco de eliminação inapropriada.

Com base nesses resultados e pesquisas futuras, alguns comportamentos inaceitáveis para os tutores podem ser evitados ou prevenidos, melhorando a relação entre tutor e animal, diminuindo o risco de abandono e transmissão de zoonoses.

Agradecimentos.- À Universidade Federal do Rio Grande do Sul pela concessão da bolsa de iniciação científica (BIC-UFRGS).

\section{REFERÊNCIAS}

Amat M., LaTorre J.L.R., Fatjó J., Mariotti V.M., VanWijk S. \& Manteca X. 2009. Potential risk factors associated with feline behavior problems. Appl. Anim. Behav. Sci. 121:134-139.

Barry K. J. \& Crowell-Davis S.L. 1999. Gender differences in the social behavior of the neutered indoor-only domestic cat. Appl. Anim. Behav. Sci. 64(6):193-211.

Beaver B.V. 2003. Feline communicative behavior and Feline eliminative behavior, p.100-126. In: Ibid. (Ed.), Feline Behavior: a guide for veterinarians. 2nd ed. Elsevier Science, USA.

Borchelt P.L. \& Voith V.L. 1986. Elimination behavior problems in cats. Compend. Contin. Educ. Pract. Vet. 8:197-207.

Borchelt P.L. \& Voith V.L. 1987. Aggressive behavior in cats. Compend. Contin. Educ. Pract. Vet. 9:50.

Borchelt P.L. \& Voith V.L. 1996. Readings in Companion Animal Behavior: veterinary learning systems. Trenton, New Jersey, p.72-80.

Calixto R. \& Justen H. 2007. Avaliação do efeito da castração e de variáveis ambientais sobre a marcação por urina e fezes em gatos (Felis catus). Acta Scient. Vet. 35(2):145-152.

Case L.P. 2010. Training and problem prevention for puppies and kittens, p.151-152. In: Ibid. (Ed.), Canine and Feline Behaviour and Training: a complete guide to understanding our two best friends. Cengage Learning, New York.

Cassey R.A. \& Bradshaw J.W.S. 2008. Owner compliance and clinical outcome measures for domestic cats undergoing clinical behavior therapy. J. Vet. Behav.: Clin. Appl. Res. 3:114-124.

Fatjó J., Ruiz de la Torre J.L. \& Manteca X. 2006. The epidemiologic of behavior problems in dogs and cats: a survey of veterinary practitioners. Animal Welfare 15:179-185.

Griffin B. \& Hume K.R. 2006. Recognition and management of stress in housed cats, p.717-734. In: August J. (Ed.), Consultations in Feline Internal Medicine. Vol.5. Elsevier, St Louis.
Hart B.L. \& Barret R.E. 1973. Effects of castration on fighting, roaming, and urine spraying in adult male cats. J. Am. Vet. Med. Assoc. 163:290-292.

Hart B.L. \& Cooper L. 1984. Factors relating to urine spraying and fighting in prepubertally gonadectomized cats. J. Am. Vet. Med. Assoc. 184:12551258.

Heath S. 2006. Problemas comportamentais comuns em felinos, p.41-56. In: Chandler E.A., Gaskell C.J. \& Gaskell R.M. (Eds), Clínica e terapêutica em felinos. Roca, São Paulo.

Heidenberger E. 1997. Housing conditions and behavioural problems of indoor cats as assessed by their owners. Appl. Anim. Behav. Sci. 52:345364.

Houpt K.A. 1985. Companion animal behavior: a review of dog and cat behavior in the field, the laboratory and the clinic. Cornell Vet. 75:248-261.

Kelsey J.L., Whittemore A.S., Evans A.S. \& Thompson W.D. 1996. Methods in Observational Epidemiology. 2nd ed. Oxford University Press, New York. 432p.

Marder A. 1997. Managing behavioral problems in puppies and kittens. Small Animal Behavior Friskes PetCare, p.15-24. (Apud Beaver 2003)

Miller D.D., Staats S.R., Partlo C. \& Rada K. 1996. Factors associated with the decision to surrender a pet to an animal shelter. J. Am. Vet. Med. Assoc. 209(4):738-742.

Neilson J. 2004. Thinking outside the box: feline elimination. J. Feline Med. Surg. 6:5-11.

Morgan M. \& Houpt K.A. 1990. Feline behavior problems: the influence of declawing. Antrozoos 3:50-53.

Overall K.L. 1997. Miscellaneous Behavioral Problems: emphasis on management, p.160-194. In: Ibid. (Ed.), Clinical Behavior Medicine for Small Animals. Mosby, St Louis.

Price E.0. 1984. Behavioral aspects of animal domestication. Quart. Rev. Biol. 59(1):1-32.

Ramos D. \& Mills D.S. 2009. Human directed aggression in Brazilian domestic cats. J. Feline Med. Surg. 11(11):835-841.

Rochlitz I. 2000. Feline welfare issues, p.208-226. In: Turner C., Bateson P. (Eds), The Domestic Cat: the biology of its behavior. University Press, Cambridge.

Souza-Dantas L.M., Soares G.M., D’Almeida J.M. \& Paixão R.L. 2009. Epidemiology of domestic cat behavioral and welfare issues: a survey of Brazilian referral animal hospitals in 2009. Int. J. Appl. Res. Vet. Med. 7(3):130-137.

Tamimi N., Malmasi A., Talebi A., Tamimi F. \& Amini A. 2015. A survey of feline behavioral problems in Tehran. Vet. Res. Forum 6(2):143-147.

Turner D.C. 1991. The ethology of behaviour problems in cats. Practitioner 13:43-50.

Wassink-Van der Schot A.A., Day C., Morton J.M., Rand J. \& Phillips C.J.C. 2016. Risk factors of behavior problems in cats presented to an Australian companion animal behavior clinic. J. Vet. Behav. 14:34-40.

Wolfe R.C., Sung W. \& Crowell-Davis S.L. 1997. Individual and gender preferences in association in a free-ranging population of domestic cats. J. Am. Vet. Soc. Anim. Behav. 19(2):4. 